\title{
Biomarkers for the Diagnosis of Acute Kidney Injury
}

\author{
Sushrut S. Waikar Joseph V. Bonventre \\ Renal Division, Department of Medicine, Brigham and Women's Hospital, Harvard Medical School, Boston, Mass., USA
}

\section{Key Words}

Acute kidney injury · Acute renal failure - Biomarker • Diagnosis

\begin{abstract}
The identification of acute kidney injury relies on tests like blood urea nitrogen and serum creatinine that were identified and incorporated into clinical practice several decades ago. This review summarizes clinical studies of newer biomarkers that may permit earlier and more accurate identification of acute kidney injury. The urine may contain sensitive and specific markers of kidney injury that are present due to either impaired tubular reabsorption and catabolism of filtered molecules or release of tubular cell proteins in response to ischemic or nephrotoxic injury. Many potential markers have been studied. Promising injury markers in the urine include $\mathrm{N}$-acetyl- $\beta-D-$ glucosaminidase, neutrophil gelatinase-associated lipocalin, kidney injury molecule-1, and interleukin-18. New biomarkers of kidney injury hold the promise of substantially improving the diagnostic approach to acute kidney injury. Adequately powered clinical studies of multiple biomarkers are needed to qualify the biomarkers before they can be fully adopted in clinical practice. Once adopted, more sensitive biomarkers of acute kidney injury hold the potential to transform the care of patients with renal disease.

Copyright $\odot 2008$ S. Karger AG, Basel
\end{abstract}

\section{Introduction}

Acute renal failure, now termed acute kidney injury (AKI), is an increasingly common and devastating complication in hospitalized patients. The current diagnostic tests for AKI include serum creatinine (SCr) and blood urea nitrogen (BUN), two biomarkers that were identified and incorporated into clinical practice several decades ago. It is now widely appreciated that SCr and BUN are suboptimal markers for AKI, and that more sensitive, specific, and early biomarkers are needed. This review sets out to cover recent developments in the field of AKI biomarker validation in clinical studies.

\section{Injury versus Failure: Towards a Troponin for the Kidney}

The new term 'acute kidney injury' instead of 'acute renal failure' - by its replacement of the word 'injury' for 'failure' - hints towards a paradigm shift in nephrology. The diagnosis of 'failure' refers to the kidney's inability to perform one of its major functions, namely glomerular filtration. The diagnosis of glomerular filtration failure is made only after endogenous filtration markers (BUN or SCr) have accumulated in the blood, typically hours or even days after an inciting event. The diagnosis of 'injury', by contrast, does not presuppose a reduction in glomerular filtration. Newer biomarkers are needed to identify correlates of cellular (typically tubular) injury, which may be present well before or in the absence of a reduction in GFR. The analogy to cardiology may be instructive: clinicians diagnosing acute myocardial infarction do not wait until a reduction in cardiac output, but rather make the diagnosis of myocardial injury on the basis of elevations of tissuespecific biomarkers in the serum.

The biological response of kidney tissue to ischemic or nephrotoxic injury may be utilized as early indicators of

\section{KARGER}

Fax +41613061234

E-Mail karger@karger.ch

www.karger.com
(C) 2008 S. Karger AG, Basel

$1660-2110 / 08 / 1094-0192 \$ 24.50 / 0$

Accessible online at:

www.karger.com/nec
Sushrut S. Waikar, MD, MPH

Brigham and Women's Hospital, MRB-4

75 Francis Street

Boston, MA 02115 (USA)

Tel. +1 617732 6020, Fax +1 617732 6392, E-Mail swaikar@partners.org 
AKI. Urine is readily accessible and has been proposed to be a more promising biological fluid than serum or plasma to identify the earliest markers of kidney injury. Time honored tests like urinary microscopy, urine osmolality, and fractional excretion of sodium or urea are nonspecific and insensitive, although careful and large prospective studies have not been conducted [1]. The urine may contain more sensitive and specific markers of kidney injury that are present due to impaired tubular reabsorption and catabolism of filtered molecules, release of enzymes or exosomes from tubular cells, and as a response of tubular cells to ischemic or nephrotoxic injury. This review will focus on several promising urinary biomarkers under investigation.

\section{Tubular Enzymes and Markers of Tubular Dysfunction}

The apical surface of proximal tubular epithelial cells contains numerous microvilli that form the brush border and contain proteins with enzymatic functions to carry out the specialized tasks of the proximal tubule. Intracellular enzymes can be released into the urine with injury [2] either by exocytosis or leakage. The proteins can exist in the free form or may be membrane-encased as exosomes. Several different classes of enzymes can be found: lysosomal proteins, such as $\mathrm{N}$-acetyl- $\beta$-D-glucosaminidase (NAG), brush border enzymes including gamma-glutamyl transferase (GGT) and alkaline phosphatase, as well as cytosolic proteins such as $\alpha$-glutathione S-transferase ( $\alpha$-GST). Furthermore, when proximal tubular epithelial cells are injured, they may not metabolize cystatin C properly, and filtered intact cystatin C may appear in the urine. Similarly, injured cells may not completely reabsorb low-molecularweight proteins that are freely filtered into the urinary space, such as $\alpha_{1^{-}}$and $\beta_{2}$-microglobulin.

Westhuyzen et al. [3] compared the predictive value of a number of tubular enzymes for the subsequent development of AKI, defined as a $50 \%$ rise in serum creatinine to at least $1.7 \mathrm{mg} / \mathrm{dl}$. Four of 26 subjects developed AKI; baseline levels of GGT, AP, NAG, $\alpha$-GST and $\pi$-GST were higher in those who developed AKI, compared to those who did not. $\alpha$-GGT and $\pi$-GST had the best predictive value on their own, with areas under the receiver-operating characteristic curve (AUC-ROC) of 0.95 (95\% CI 0.79-1.0) and 0.93 (95\% CI 0.74-1.0), respectively. Changes in enzyme levels preceded detectable changes in timed creatinine clearance. However, when the authors attempted to develop cutpoints based on this small study and tested the generalizability of their results in a test population of $19 \mathrm{pa}-$ tients (4 of whom developed AKI), the sensitivity and specificity of these biomarkers were significantly reduced.
Several investigators have examined the ability of tubular enzymes to predict adverse clinical outcomes. Herget-Rosenthal et al. [4] risk-stratified patients with nonoliguric AKI (defined as a doubling in creatinine from a baseline concentration of $<106 \mu \mathrm{mol} / \mathrm{l}$ to at least 115 $\mu \mathrm{mol} / \mathrm{l})$ using tubular enzymes as biomarkers. They identified 73 subjects who met prespecified criteria for AKI; 26 of these individuals subsequently required dialysis. They measured urinary excretion of cystatin C, $\alpha_{1^{-}}$and $\beta_{2}$-microglobulin, $\alpha$-GST, NAG, retinol-binding protein (RBP), GGT and lactate dehydrogenase on the day of study enrollment. Cystatin C and $\alpha_{1}$-microglobulin (markers of abnormal proximal tubule function) had the best predictive value for the need for dialysis, with AUC-ROC curves of 0.92 and 0.86 , respectively. Of the tubular enzymes studied, NAG had the best predictive value, with an AUC-ROC of 0.81. In another study, Chew et al. [5] found that levels of NAG and tissue non-specific alkaline phosphatase were higher in AKI patients with poor outcomes (defined as need for dialysis or death). Liangos et al. [6] recently performed a study of NAG and kidney injury molecule-1 (KIM-1, a tubular injury marker, discussed below) in 201 patients with established AKI. They found that elevated NAG levels portended poor clinical outcomes, with the odds of death or dialysis requirement increased over fivefold in patients with the highest versus lowest quartiles or urinary NAG levels, even after careful multivariable adjustment for disease severity and comorbidity. The predictive power of KIM-1 levels in this study will be discussed below.

Tubular enzymes present in the urine have long been studied as markers of AKI, but they have not been adopted in widespread clinical use either as early diagnostic tests, prognostic indicators, or surrogate endpoints for interventional studies. Some authors have suggested that tubular enzymes are overly sensitive, because they tend to rise after injuries such as cardiopulmonary bypass without an associated rise in SCr $[7,8]$. Investigators should exercise caution, however, in interpreting performance characteristics of new biomarkers against a gold standard like SCr that has poor specificity and sensitivity: cardiac troponin would appear to be nonspecific against earlier (and now discredited) cardiac biomarkers like lactate dehydrogenase.

\section{Neutrophil Gelatinase-Associated Lipocalin (NGAL)}

NGAL is one of the best-studied urinary biomarkers of AKI to date. Also known as lipocalin-2 or siderocalin, NGAL was first discovered as a protein in granules of hu- 
man neutrophils; animal studies showed its promise as an early marker of ischemic and nephrotoxic kidney injury [9].

Mishra et al. [10] prospectively obtained serial urine and serum samples from 71 children undergoing cardiopulmonary bypass for surgical correction of congenital heart disease. Twenty children developed AKI, defined as a $50 \%$ increase in SCr. Urinary NGAL at just $2 \mathrm{~h}$ following $\mathrm{CPB}$ almost perfectly predicted which patients would go on to develop AKI (AUC-ROC 0.998). Serum NGAL was inferior to urinary NGAL for the identification of AKI. As encouraging as these results were, it should be noted that $29 \%$ of eligible patients were excluded due to perioperative use of ibuprofen, angiotensin-converting enzyme inhibitors, gentamicin, or vancomycin. A larger follow-up study of 120 children (using similar exclusion criteria) by Dent et al. [11] showed that 2-hours postoperative serum NGAL was predictive of AKI (AUC-ROC 0.96) and correlated with postoperative change in $\mathrm{SCr}$, duration of $\mathrm{AKI}$, and length of stay.

Wagener reported less sanguine results on urinary NGAL in 81 adult patients undergoing cardiac surgery; the only exclusion criterion was pre-existing end-stage renal disease [12]. Sixteen patients developed AKI, defined as a $50 \%$ increase in SCr. Urinary NGAL levels were consistently higher immediately postoperatively and at $1,3,18$, and $24 \mathrm{~h}$ postoperatively in patients who went on to develop AKI. Substantial overlap, however, was noted between patients who did and did not develop AKI. The AUC-ROC for NGAL ranged from 0.67 (immediately after surgery) to 0.80 (18 h following surgery). The salient differences between the pediatric and adult cardiac surgery cohorts included patient age, comorbidity, and exclusion criteria.

Parikh et al. [13] studied urinary NGAL in 53 consecutive patients undergoing living or deceased donor kidney transplantation. NGAL levels (normalized to urine creatinine concentration) were significantly higher in deceased donor recipients with delayed graft function (DGF) ( $\mathrm{n}=$ 10, median 3,306 $\mathrm{ng} / \mathrm{mg}$ creatinine) than prompt graft function ( $\mathrm{n}=20$, median $756 \mathrm{ng} / \mathrm{mg}$ creatinine). A cutoff value of $1,000 \mathrm{ng} / \mathrm{mg}$ creatinine had $90 \%$ sensitivity and $83 \%$ specificity for the identification of DGF; the AUCROC was 0.90 .

Zappitelli et al. [15] studied urinary NGAL in 140 children admitted to the intensive care unit requiring mechanical ventilation. Urine was collected daily for 4 days. The authors found on cross-sectional analysis that mean and peak urinary NGAL levels were higher in patients with worsening degrees of AKI (as judged by the pediatric RIFLE criteria). At $48 \mathrm{~h}$ prior to the development of AKI, urinary NGAL had an AUC-ROC of 0.79 for the subsequent development of AKI.

Urinary NGAL has also been studied as a surrogate marker of kidney injury from aprotinin, a nephrotoxic fibrinolytic used in cardiac surgery. Wagener et al. [36] found that postoperative levels of urinary NGAL were almost 20 times higher in patients who received aprotinin compared to epsilon amino-caproic acid, lending support to the suggestion of aprotinin's nephrotoxicty. Urinary NGAL was also found to be higher after coronary angiography in 13 patients with AKI than in 27 controls without AKI [17].

\section{Interleukin-18}

IL-18 was found to potentiate ischemic AKI and to be detectable in the urine of mice subjected to ischemic kidney injury [18]. Urinary IL-18 has been studied by Parikh and coworkers in a variety of clinical settings, including delayed graft function [14], cardiac surgery [19], acute respiratory distress syndrome [20], and cross-sectionally in patients with and without kidney disease [21].

The first AKI study of urinary IL-18 in humans was a cross-sectional comparison of patients with ATN $(n=14)$, pre-renal azotemia $(\mathrm{n}=8)$, UTI $(\mathrm{n}=5)$, CKD $(\mathrm{n}=12)$, transplant recipients $(n=22)$, and healthy controls $(n=11)$ [21]. The highest levels of urinary IL-18 were observed in patients with ATN and delayed graft function, with relatively little overlap from patients with pre-renal azotemia, urinary tract infections, and CKD. The AUC-ROC from this cross-sectional cohort (for the identification of ATN, including delayed graft function) was 0.95 , with a sensitivity of $85 \%$ and specificity of $88 \%$ at a cutoff of 500 pg IL$18 /$ mg creatinine.

The NIH sponsored Acute Respiratory Distress Syndrome Network trial of low versus high tidal volume ventilation was the source of urine samples in a subsequent study of urinary IL-18 [20]. Parikh and colleagues performed a prospective, nested, case-control study in 138 of the 861 patients enrolled; exclusion criteria included a baseline SCr $>1.2 \mathrm{mg} / \mathrm{dl}$. They found that urinary IL-18 levels were higher in those patients who developed AKI (defined as a 50\% increase in SCr within 6 days of enrollment), and that higher levels were predictive of mortality. The AUC-ROC for IL-18 (not normalized to urine creatinine) was 0.73 at $24 \mathrm{~h}$ prior to AKI diagnosis; this value does not compare favorably with the AUC-ROC of 0.95 from the cross-sectional study of urinary IL-18.

Parikh et al. [20] also measured IL-18 in urine samples collected in the pediatric cardiac surgery cohort used to 
study NGAL. They measured IL-18 in all 20 cases of AKI and in 35 of the 51 non-AKI cases (matched according to race, gender, and age to AKI cases). Compared to NGAL, which increased 25 -fold within $2 \mathrm{~h}$ and declined after $6 \mathrm{~h}$ of CPB, IL-18 increased at $4-6 \mathrm{~h}$ and remained elevated up to $48 \mathrm{~h}$ following CPB. The reported AUC-ROCs for IL-18 were 0.61 at $4 \mathrm{~h}, 0.75$ at $12 \mathrm{~h}$, and 0.73 at $24 \mathrm{~h}$, substantially lower than the 0.998 reported by Mishra for NGAL at $2 \mathrm{~h}$ following $\mathrm{CPB}$.

IL-18 was also studied by Washburn et al. [14] in critically ill children requiring mechanical ventilation (identical cohort as studied by Zappitelli et al. [15] for NGAL). They found on cross-sectional analysis that peak urinary IL-18 levels were higher in patients with worsening degrees of AKI (as judged by the pediatric RIFLE criteria). However, in prospective analysis IL-18 demonstrated no ability to predict the subsequent development of AKI (AUC-ROC $0.54)$. Not surprisingly for a pro-inflammatory cytokine that plays an important role in sepsis, urinary IL-18 was significantly higher in patients with sepsis than in those without, and limited its diagnostic ability for the early identification of AKI in this cohort.

Urinary IL-18 has also been studied as a biomarker of contrast nephropathy with mixed results. Ling et al. [17] found higher urinary levels in 13 patients who developed AKI following coronary angiography than in 27 non-AKI controls ( $\mathrm{p}<0.01$ ), whereas Bulent et al. [22] found no difference in a similar number of patients with and without AKI.

\section{$\mathrm{Na}^{+} / \mathrm{H}^{+}$Exchanger Isoform 3 (NHE3)}

NHE3 is the most abundant sodium transporter in the renal tubule and is responsible for proximal reabsorption of up to $70 \%$ of filtered sodium and bicarbonate. Du Cheyron et al. [23] performed a cross-sectional study of 68 patients admitted to the ICU. They isolated membrane fractions from the urine and measured NHE3 concentrations using semiquantitative immunoblotting. NHE3 protein was undetectable in patients without AKI $(\mathrm{n}=14)$, detectable at relatively low levels in patients with pre-renal azotemia $(n=17)$ and post-renal obstruction $(n=3)$, and was significantly elevated in ATN ( $\mathrm{n}=17 ; 6.6$-fold higher than in pre-renal azotemia). The same investigators also measured urinary retinol-binding protein (RBP), the primary plasma transport protein for vitamin A which is filtered by the glomerulus and then reabsorbed by the proximal tubule. Urinary RBP was significantly higher in patients with ATN than normal controls, but significant overlap was noted, particularly with pre-renal azotemia.

\section{Kidney Injury Molecule-1 (KIM-1)}

Kidney injury molecule-1 ('KIM-1' in humans, or 'Kim-1' in rodents) was identified as the single most upregulated gene in postischemic rat kidney using a PCR-based technique [24]. KIM-1 encodes a type I cell membrane glycoprotein containing, in its extracellular portion, a novel sixcysteine immunoglobulin-like domain and a threonine/ serine and proline-rich domain characteristic of mucinlike O-glycoslyated proteins, suggesting its potential involvement in cell-cell and/or cell-matrix interactions [25]. After proximal tubular kidney injury, the ectodomain of KIM-1 protein is shed from cells into the urine in rodents and in humans. In both ischemia-reperfusion and cisplatin-induced nephrotoxicity models in the rat, urinary Kim-1 is a sensitive and specific indicator of proximal tubular kidney injury and is increased earlier than any of the conventional biomarkers, e.g. plasma creatinine, blood urea nitrogen, glycosuria, proteinuria, and urinary NAG [26]. In recently completed studies of 8 mechanistically different proximal tubule nephrotoxicants and 2 different hepatotoxicants in rats, Kim-1 had an AUC-ROC of 0.99 for proximal tubular toxicity, using histopathology as the gold standard; of 21 urinary markers studied, Kim-1 was found to be to have the highest sensitivity and specificity [27].

Human studies have begun to confirm the promise of urinary KIM-1 for the diagnosis of AKI. Han et al. [28] demonstrated marked expression of KIM-1 in kidney biopsy specimens from 6 patients with acute tubular necrosis (ATN), and found elevated urinary levels of KIM-1 in 7 patients with ischemic ATN; urinary levels of KIM-1 were significantly lower in contrast nephropathy $(n=7)$, although the levels did correlate with severity of contrastinduced injury. Levels of urinary KIM-1 were lower in AKI not due to ATN $(n=9), \operatorname{CKD}(n=9)$, and were below limits of detection in normal subjects $(\mathrm{n}=8)$ [28]. KIM-1 is also highly expressed in the setting of renal cell carcinoma (RCC), as shown by Han et al. [29]. KIM-1 was detectable in the urine of patients with RCC, suggesting a potential role for early non-invasive diagnosis. Lin et al. [30] studied KIM-1 protein expression in a large series of 480 neoplasms and found KIM-1 expression in papillary, clear cell, and metastatic RCC but not chromophobe RCC. They also demonstrated KIM-1 staining in 15 of 16 cases of clear cell carcinoma of the ovary. Van Timmeren et al. [31] stained for KIM-1 protein in tissue specimens from 102 patients who underwent kidney biopsy for a variety of kidney diseases and 7 patients who underwent nephrectomy for renal cell carcinoma. No tissue KIM-1 was found in patients with minimal change disease or in the 
tumor-free samples of renal cell carcinoma. In all other disease conditions, KIM-1 protein was identified in dedifferentiated proximal tubular cells and correlated with tubulointerstitial fibrosis and inflammation. In the subset of patients who underwent urine collection near the time of biopsy, urinary KIM-1 levels correlated with tissue expression of KIM-1. Urinary KIM-1 may therefore hold promise as a non-invasive assessment of the activity and prognosis of a variety of acute and chronic kidney diseases. The diagnostic and prognostic role of KIM-1 in kidney transplant recipients was evaluated by Zhang et al. [32] in 25 protocol biopsies, 25 biopsies of patients with active tubular injury, and 12 patients with acute cellular rejection. Focal KIM-1 expression was found in $28 \%$ of protocol biopsies despite the absence of conventional histologic evidence of tubular cell injury. Proximal tubule KIM-1 expression was found in all patients with histologic evidence of tubular cell injury, and higher KIM-1 staining correlated with improved renal outcomes at 18 months. Van Timmeren et al. [33] studied 24-hour urinary KIM-1 excretion in 145 stable renal transplant recipients who were subsequently followed for 4 years. Higher KIM-1 excretion was associated with significantly higher risk of graft loss over the follow-up period. High KIM-1 excretion was also associated with proteinuria, low creatinine clearance, and high donor age, but was independently associated with graft loss after multivariate adjustment for these variables.

Liangos et al. [6] studied urinary KIM-1 (and NAG, see above) at the time of nephrology consultation in $201 \mathrm{pa}-$ tients with established AKI. Because non-AKI controls were not included in this study, diagnostic performance characteristics like sensitivity, specificity, or the area under the receiver operating characteristics curve were not reported. KIM-1 demonstrated prognostic significance: elevated levels were significantly associated with the clinical composite endpoint of death or dialysis requirement, even after adjustment for disease severity or comorbidity. How KIM-1 and other markers will compare to other predictive markers and clinical scoring systems is the subject

pilot study of patients $(n=17)$ undergoing kidney transplantation, and found markedly higher levels among those with DGF following deceased donor transplantation. Zhou et al. [35] have focused on urinary exosomes (small excreted vesicles which contain membrane and cytosolic proteins) as a potential source of novel AKI biomarkers. Exosomal fetuin-A was identified in preclinical rodent models of ischemic and cisplatin-induced AKI; a small pilot study in 9 humans showed exosomal fetuin-A to be present in the urine of ICU patients with AKI but not in healthy volunteers or ICU patients without AKI [35].

\section{Conclusion}

New biomarkers under clinical investigation will likely perform differently with respect to disease specificity (e.g. sepsis vs. nephrotoxic versus postoperative AKI), time course (early vs. late markers), and prognostic characteristics (markers of incipient AKI vs. markers of prognosis in established AKI). Establishing the optimal test(s) for a given clinical scenario will require prospective validation in large numbers of patients with a variety of causes of AKI, preferably with measurement of numerous candidate biomarkers for the purpose of efficiency. The possibility that new biomarkers may be superior to SCr for the identification of AKI will require investigators to test the creatinineindependent associations between biomarker levels and exposures (e.g. cardiopulmonary bypass time, dose of nephrotoxin administration) and outcomes (e.g. not only AKI as defined by creatinine but also length of stay, need for dialysis, and mortality). Early and accurate diagnosis of AKI will allow interventional studies to be performed in a timely fashion, which is a prerequisite for the future development of effective prevention and therapeutic strategies that have eluded nephrology for years. of extensive ongoing studies.

\section{Other Markers}

Keratinocyte-derived chemokine was found by Molls et al. [34] in a mouse model of renal ischemia-reperfusion injury to be elevated in serum and urine $3 \mathrm{~h}$ following injury. These investigators measured urinary levels of a structurally homologous molecule in humans, termed human growth-related oncogene- $\alpha$ (Gro- $\alpha)$, in a small

\section{References}

1 Bagshaw SM, Langenberg C, Bellomo R: Urinary biochemistry and microscopy in septic acute renal failure: a systematic review. Am J Kidney Dis 2006;48:695-705.

2 D’Amico G, Bazzi C: Urinary protein and enzyme excretion as markers of tubular damage. Curr Opin Nephrol Hypertens 2003;12: 639-643.

- 3 Westhuyzen J, Endre Z, Reece G, Reith D, Saltissi D, Morgan T: Measurement of tubular enzymuria facilitates early detection of acute renal impairment in the intensive care unit. Nephrol Dial Transplant 2003;18:543551. 
4 Herget-Rosenthal S, Poppen D, Husing J, Marggraf G, Pietruck F, Jakob H-G, Phillipp T, Kribben A: Prognostic value of tubular proteinuria and enzymuria in nonoliguric acute tubular necrosis. Clin Chem 2003;50: 552-558.

5 Chew SL, Lins RL, Daelemans R, Nuyts GD, De Broe ME: Urinary enzymes in acute renal failure. Nephrol Dial Transplant 1993;8: 507-511.

-6 Liangos O, Perianayagam MC, Vaidya VS, Han WK, Wald R, Tighiouart H, MacKinnon RW, Li L, Balakrishnan VS, Pereira BJ, Bonventre JV, Jaber BL: Urinary N-acetylbeta- $(D)$-glucosaminidase activity and kidney injury molecule-1 level are associated with adverse outcomes in acute renal failure. J Am Soc Nephrol 2007;18:904-912.

-7 Eijkenboom JJ, van Eijk LT, Pickkers P, Peters WH, Wetzels JF, van der Hoeven HG: Small increases in the urinary excretion of glutathione S-transferase A1 and P1 after cardiac surgery are not associated with clinically relevant renal injury. Intensive Care Med 2005;31:664-667.

8 Hamada Y, Kanda T, Anzai T, Kobayashi I, Morishita Y: N-acetyl-beta-D-glucosaminidase is not a predictor, but an indicator of kidney injury in patients with cardiac surgery. J Med 1999;30:329-336.

\9 Mishra J, Ma Q, Prada A, Mitsnefes M, Zahedi K, Yang J, Barasch J, Devarajan P: Identification of neutrophil gelatinase-associated lipocalin as a novel early urinary biomarker for ischemic renal injury. J Am Soc Nephrol 2003;14:2534-2543.

10 Mishra J, Dent C, Tarabishi R, et al: Neutrophil gelatinase-associated lipocalin (NGAL) as a biomarker for acute renal injury after cardiac surgery. Lancet 2005;365:1231-1238.

- 11 Dent CL, Ma Q, Dastrala S, Bennett M, Mitsnefes MM, Barasch J, Devarajan P: Plasma neutrophil gelatinase-associated lipocalin predicts acute kidney injury, morbidity and mortality after pediatric cardiac surgery: a prospective uncontrolled cohort study. Crit Care 2007;11:R127.

-12 Wagener G, Jan M, Kim M, Mori K, Barasch JM, Sladen RN, Lee HT: Association between increases in urinary neutrophil gelatinaseassociated lipocalin and acute renal dysfunction after adult cardiac surgery. Anesthesiology 2006;105:485-491.

13 Parikh CR, Jani A, Mishra J, Ma Q, Kelly C, Barasch J, Edelstein CL, Devarajan P: Urine NGAL and IL-18 are predictive biomarkers for delayed graft function following kidney transplantation. Am J Transplant 2006;6: 1639-1645.

14 Washburn KK, Zappitelli M, Arikan AA, Loftis L, Yalavarthy R, Parikh CR, Edelstein CL, Goldstein SL: Urinary interleukin-18 is an acute kidney injury biomarker in critically ill children. Nephrol Dial Transplant 2008;23:566-572.
15 Zappitelli M, Washburn KK, Arikan AA, Loftis L, Ma Q, Devarajan P, Parikh CR, Goldstein SL: Urine neutrophil gelatinaseassociated lipocalin is an early marker of acute kidney injury in critically ill children: a prospective cohort study. Crit Care 2007; 11:R84.

16 Shaw AD, Stafford-Smith M, White WD, et al: The effect of aprotinin on outcome after coronary-artery bypass grafting. N Engl J Med 2008;358:784-793.

17 Ling W, Zhaohui N, Ben H, Leyi G, Jianping L, Huili D, Jiaqi Q: Urinary IL-18 and NGAL as early predictive biomarkers in contrastinduced nephropathy after coronary angiography. Nephron Clin Pract 2008;108:c176c181.

-18 Melnikov VY, Ecder T, Fantuzzi G, Siegmund B, Lucia MS, Dinarello CA, Schrier RW, Edelstein CL: Impaired IL-18 processing protects caspase-1-deficient mice from ischemic acute renal failure. J Clin Invest 2001;107:1145-1152.

19 Parikh CR, Mishra J, Thiessen-Philbrook H, Dursun B, Ma Q, Kelly C, Dent C, Devarajan P, Edelstein CL: Urinary IL-18 is an early predictive biomarker of acute kidney injury after cardiac surgery. Kidney Int 2006;70:199203.

20 Parikh CR, Abraham E, Ancukiewicz M, Edelstein CL: Urine IL-18 is an early diagnostic marker for acute kidney injury and predicts mortality in the intensive care unit. J Am Soc Nephrol 2005;16:3046-3052.

21 Parikh CR, Jani A, Melnikov VY, Faubel S, Edelstein CL: Urinary interleukin-18 is a marker of human acute tubular necrosis. Am J Kidney Dis 2004;43:405-414.

22 Bulent Gul CB, Gullulu M, Oral B, Aydinlar A, Oz O, Budak F, Yilmaz Y, Yurtkuran M: Urinary IL-18: a marker of contrast-induced nephropathy following percutaneous coronary intervention? Clin Biochem 2008;41: 544-547.

23 du Cheyron D, Daubin C, Poggioli J, Ramakers M, Houillier P, Charbonneau P, Paillard M: Urinary measurement of $\mathrm{Na}^{+} / \mathrm{H}^{+} \mathrm{ex}-$ changer isoform 3 (NHE3) protein as new marker of tubule injury in critically ill patients with ARF. Am J Kidney Dis 2003;42: 497-506.

24 Ichimura T, Bonventre JV, Bailly V, Wei H, Hession CA, Cate RL, Sanicola M: Kidney injury molecule-1 (KIM-1), a putative epithelial cell adhesion molecule containing a novel immunoglobulin domain, is up-regulated in renal cells after injury. J Biol Chem 1998;273:4135-4142.

25 Bailly V, Zhang Z, Meier W, Cate R, Sanicola $\mathrm{M}$, Bonventre JV: Shedding of kidney injury molecule-1, a putative adhesion protein involved in renal regeneration. J Biol Chem 2002;277:39739-39748.
26 Vaidya VS, Ramirez V, Ichimura T, Bobadilla NA, Bonventre JV: Urinary kidney injury molecule-1: a sensitive quantitative biomarker for early detection of kidney tubular injury. Am J Physiol Renal Physiol 2006;290: F517-F529.

27 Dieterle F, Staedtler F, Grenet O, et al: Qualification of biomarkers for regulatory decision making: a kidney safety biomarker project (abstract, Society of Toxicology). Toxicologist 2007;96:383.

28 Han WK, Bailly V, Abichandani R, Thadhani R, Bonventre JV: Kidney injury molecule-1 (KIM-1): a novel biomarker for human renal proximal tubule injury. Kidney Int 2002;62:237-244.

-29 Han WK, Alinani A, Wu CL, Michaelson D, Loda M, McGovern FJ, Thadhani R, Bonventre JV: Human kidney injury molecule-1 is a tissue and urinary tumor marker of renal cell carcinoma. J Am Soc Nephrol 2005;16:11261134.

-30 Lin F, Zhang PL, Yang XJ, Shi J, Blasick T, Han WK, Wang HL, Shen SS, Teh BT, Bonventre JV: Human kidney injury molecule-1 (hKIM-1): a useful immunohistochemical marker for diagnosing renal cell carcinoma and ovarian clear cell carcinoma. Am J Surg Pathol 2007;31:371-381.

31 van Timmeren MM, van den Heuvel MC, Bailly V, Bakker SJ, van Goor H, Stegeman CA: Tubular kidney injury molecule-1 (KIM1) in human renal disease. J Pathol 2007;212: 209-217.

32 Zhang PL, Rothblum LI, Han WK, Blasick TM, Potdar S, Bonventre JV: Kidney injury molecule-1 expression in transplant biopsies is a sensitive measure of cell injury. Kidney Int 2008;73:608-614.

-33 van Timmeren MM, Vaidya VS, van Ree RM, Oterdoom LH, de Vries AP, Gans RO, van Goor H, Stegeman CA, Bonventre JV, Bakker SJ: High urinary excretion of kidney injury molecule-1 is an independent predictor of graft loss in renal transplant recipients. Transplantation 2007;84:1625-1630.

- 34 Molls RR, Savransky V, Liu M, Bevans S, Mehta T, Tuder RM, King LS, Rabb H: Keratinocyte-derived chemokine is an early biomarker of ischemic acute kidney injury. Am J Physiol Renal Physiol 2006;290:F1187F1193.

35 Zhou H, Pisitkun T, Aponte A, et al: Exosomal fetuin-A identified by proteomics: a novel urinary biomarker for detecting acute kidney injury. Kidney Int 2006;70:18471857.

36 Wagener G, Gubitosa G, Wang S, Borregaard $\mathrm{N}$, Kim M, Lee HT: Increased incidence of acute kidney injury with aprotinin use during cardiac surgery detected with urinary NGAL. Am J Nephrol 2008;28:576-582. 